\title{
Article
}

\section{Qualitative undergraduate project supervision in psychology: Current practices and support needs of supervisors across North East England and Scotland}

Wiggins, S, Gordon-Finlayson, A, Becker, S and Sullivan, Cath Available at https://clok.uclan.ac.uk/12513/

Wiggins, S, Gordon-Finlayson, A, Becker, S and Sullivan, Cath orcid iconORCID: 0000-0001-5417-8945 (2015) Qualitative undergraduate project supervision in psychology: Current practices and support needs of supervisors across North East England and Scotland. Qualitative Research in Psychology . ISSN 1478-0887

It is advisable to refer to the publisher's version if you intend to cite from the work. http://dx.doi.org/10.1080/14780887.2015.1075641

For more information about UCLan's research in this area go to http://www.uclan.ac.uk/researchgroups/ and search for < name of research Group>.

For information about Research generally at UCLan please go to http://www.uclan.ac.uk/research/

All outputs in CLoK are protected by Intellectual Property Rights law, including Copyright law. Copyright, IPR and Moral Rights for the works on this site are retained by the individual authors and/or other copyright owners. Terms and conditions for use of this material are defined in the policies page. 


\title{
Qualitative undergraduate project supervision in psychology: Current practices and support needs of supervisors across North East England and Scotland ${ }^{1}$
}

\author{
Sally Wiggins ${ }^{1}$, Alasdair Gordon-Finlayson ${ }^{2}$, Sue Becker ${ }^{3}$, Cath Sullivan ${ }^{4}$ \\ ${ }^{1}$ University of Strathclyde, Glasgow, UK, ${ }^{2}$ University of Northampton, Northampton, UK, \\ ${ }^{3}$ Teeside University, Middlesbrough, UK, ${ }^{4}$ University of Central Lancashire, Preston, UK
}

Corresponding author: Dr Sally Wiggins, School of Psychological Sciences and Health, Graham Hills Building, 40 George Street, Glasgow, G1 1QE. Email: sally.wiggins@strath.ac.uk.

\begin{abstract}
:
The dissertation is a core component of a psychology undergraduate degree, though very little research has been conducted into supervision processes at undergraduate level. This study examined the accounts of supervisors of qualitative dissertations, in order to identify current practices of supervision and possible resources that might support supervision. Seventeen supervisors from psychology departments in North East England and Scotland were interviewed and three main themes were identified using thematic analysis: the quantitative culture in psychology teaching, supervisors' expertise, and the supervision process. Supervisors noted that students were typically constrained in their choice of methodology due to limited qualitative methods teaching, lack of training and guidance for supervisors, and concerns about the risks of demanding qualitative projects. Supervisors therefore often reported staying within their comfort zone, electing where possible to supervise only the methods that they themselves use.

Recommendations for practical resources are provided to help support students and supervisors in the process of undertaking qualitative psychology dissertations.
\end{abstract}

Keywords: qualitative research, undergraduate dissertation, research methods teaching, empirical project, supervision.

\footnotetext{
${ }^{1}$ This is an Accepted Manuscript of an article published by Taylor \& Francis in Qualitative Research in Psychology on 24.7.15, available online: http://www.tandfonline.com/doi/full/10.1080/14780887.2015.1075641.
} 


\section{Biographical notes}

Sally Wiggins is a Senior Lecturer in Psychology at the University of Strathclyde, Glasgow, UK. Her research focuses on eating practices and processes - such as food preferences, satiety and disgust - and how these are managed discursively in everyday interaction. She is also interested in qualitative methods teaching and problem-based learning.

Alasdair Gordon-Finlayson is a Senior Lecturer in Psychology at the University of Northampton, UK. His research interests include identity change in religious contexts, mindfulness, and the teaching of qualitative research methods in psychology, and he is interested in constructivist applications of grounded theory methodology.

Sue Becker is a Senior Lecturer in Psychology at Teeside University. She teaches qualitative research methods and has particular interests in visual methodologies and semiotic analysis. Her research interests focus on humour in health-care interactions and joke-telling in stand-up comedy.

Cath Sullivan is a Senior Lecturer in Psychology at the University of Central Lancashire, Preston, UK. She teaches qualitative research methods to undergraduate and postgraduate students and, along with her co-authors, has been developing resources and training to support lecturers teaching qualitative methods to psychology students for over 10 years. Cath's research interests are mainly focused on social psychology, gender and mindfulness. 


\section{Qualitative undergraduate project supervision in psychology: Current practices and support needs of supervisors across North East England and Scotland}

\section{Introduction}

Qualitative research methods training is a fundamental aspect of the skills training that prepares psychology graduates for employment and further learning, and a key element of this is the final year dissertation. Increasingly, psychology research methods teaching across the UK includes both quantitative and qualitative methods, and lecturers need to be able to facilitate students' learning of qualitative methods even if they do not themselves use these methods (Forrester \& Koutsopoulou, 2008). Guidance for the supervision of qualitative dissertations is relatively scarce, however, as is research into supervision practices and processes within psychology departments. This paper examines the accounts of psychology lecturers in universities across North East England and Scotland in relation to supervising qualitative undergraduate dissertations. In doing so, it contributes to the literature on the supervision of qualitative psychology (Gough, Lawton, Madill \& Stratton, 2003; Madill, Gough, Lawton \& Stratton, 2005; Shaw, Dyson \& Peel, 2008) and addresses a key area of professional development in psychology research methods training.

The importance of developing graduates’ qualitative research skills and supporting the supervision of qualitative dissertations is acknowledged by the Quality Assurance Agency (QAA, 2010) and the Economic and Social Research Council (ESRC, 2004) in the UK, and qualitative methods are a required element of UK undergraduate psychology degrees (BPS, 2014; QAA, 2010). Psychology graduates are expected to have skills in the basic research 
methods of the discipline and this includes qualitative methods. Equipping psychologists with skills in both qualitative and quantitative methods is important to allow the discipline to address a full range of research questions in the future and can also contribute to student employability. In spite of this, there are relatively few guidelines for supervising qualitative dissertations and little is known about the supervision process itself.

Central to students’ perceived satisfaction during dissertation work is their relationship with, and confidence in, their dissertation supervisor (Calvert \& Casey, 2004; Todd, Smith \& Bannister, 2006). This confidence relies on the supervisor being proficient not only in the chosen research topic or methodology, but also in their capabilities as a supervisor. The practicalities of supervising dissertations can therefore be a source of stress and anxiety (for both students and supervisors), particularly as there is little formal training in supervision (Todd et al., 2006). As the supervisory process in psychology is not routinely scrutinized in professional body accreditation, QAA reviews or in standard peer observation practices, its characterisation as an opaque process is not surprising (Rowley \& Slack, 2004). The changing role and nature of the dissertation in honours degrees - including the greater diversity of research methods from which students can choose - also increases the need to review and develop the supervisor role (Rowley \& Slack, 2004). Improving an understanding of the practices and processes of supervision will also potentially enhance students’ satisfaction with their dissertation work.

Research on academic supervision has tended to focus on learners who are beyond the undergraduate stage, for example postgraduate research students (Anderson, Day \& McLaughlin, 2006; Maxwell \& Smyth, 2010), clinical psychology trainees (Harper, O’Connor, Selt \& Stevens, 2008) and postgraduate dissertations (Shaw, Dyson and Peel, 2008; Maunder, GordonFinlayson, Callaghan \& Roberts, 2012). There have also been studies on aspects of 
undergraduate supervision, including student experiences of supervision and feedback (Heinze \& Heinze, 2009; Mills \& Matthews, 2009), topic choice and data collection issues (I’Anson \& Smith, 2004). A small number of studies have examined supervisors' experiences of supervising undergraduate projects (Todd, Smith \& Bannister, 2006) and some have focused specifically on the experience of supervising qualitative dissertations (Gough et al., 2003; Madill et al., 2005). In one such study, supervisors highlighted the "struggle with colleagues for acceptance of qualitative research on what is perceived to be an uneven playing field” (Gough, Lawton, Madill \& Stratton, 2003, p. 4). A related study by Shaw, Dyson and Peel (2008) made the similar point that supervisors may try to protect students from the dominance of quantitative methods by dissuading them from using qualitative methods, restricting their choice of methodology and carefully selecting the second marker. These studies provide a useful basis for developing supervision practice, but more research is needed to illuminate the current practices and processes of those supervising qualitative undergraduate dissertations in order to consider what resources and further support might be developed.

This is a particularly topical issue: it is only relatively recently that qualitative methods have been a compulsory part of teaching in psychology, and there are likely to be a number of staff members who require training and support in the supervision of qualitative dissertations. A decade ago, psychology lecturers supervising qualitative dissertations reported that students’ relative lack of prior training makes supervision especially demanding (Madill et al., 2005), and the situation may have changed in the intervening period. There are also many more existing resources that supervisors of quantitative dissertations can recommend to students to complement direct supervision. For example, there are a number of textbooks on quantitative dissertation work for psychology undergraduates, whereas the first dedicated textbook for 
students doing qualitative dissertations has only been available relatively recently (Sullivan, Gibson \& Riley, 2012). Similarly, the long-standing use of quantitative methods for psychology students' empirical work at all levels has created a body of well-established traditions and areas of agreed good practice supervisors could draw upon. Such a body of established good practice is only in its infancy in relation to qualitative dissertations. Provisional guidelines for the supervision of qualitative dissertations in psychology have been produced (Madill et al., 2005), and these could form a basis for further development. Since this useful starting point there has been little similar activity. Not only are there few resources specifically focused on training and support for academics supervising qualitative projects, therefore, there is also relatively little known about the type of training and support needed in this area.

In attending to this issue, the current project aims to identify the practices and processes of staff members who supervise qualitative psychology dissertations, including noting the breadth of qualitative methodologies supervised, current supervision practices, and specific needs of supervision. In addressing these issues, we aim to renew the debate in this area and to use the empirical findings to highlight practical interventions or recommendations for future practice in qualitative dissertation supervision.

\section{Methods}

\section{Data collection}

Potential participants were identified through the authors' own contacts and through asking colleagues in psychology departments to suggest staff members who may have experience of supervising qualitative dissertations. In total, seventeen participants were interviewed for this 
research project. The geographical spread of the data collection (in North East England and Scotland) was used to provide some breadth of experience across different institutions and geographical contexts in these areas. Ethical approval for the project was obtained from the institutions of the two authors who conducted the interviews (Sue Becker, Teeside University, and Sally Wiggins, University of Strathclyde). Written consent was obtained from all participants, and all identifying features have been anonymised to protect their academic identities.

With the exception of one interview (conducted by a postgraduate student with a member of the research team as the interviewee) the interviews were conducted by either Sue Becker (North East England dataset) or Sally Wiggins (Scotland dataset). The interview schedule was semi-structured and covered demographic information (such as teaching and research specialisms, length of time supervising qualitative dissertations), current and past experiences of supervision (including processes, quality assurance and any problematic issues), and the discussion of needs and resources when considering supervision in future years. The participants were also asked which methods they had supervised as part of qualitative dissertation projects. Eight of our participants used only qualitative methods in their own research, seven used a mixture of qualitative and quantitative, and two used only quantitative methods in their own research.

Table 1 here

All interviews were audio-recorded (either face-to-face or using a telephone recording device, due to the geographical spread of the participants), and transcribed to words-only level. The 
interviews typically lasted between 45 to 60 minutes. Participants were allocated numbers (P1, P2, and so on), and institutions were also anonymised to reduce the possibility of participants being identified. As there are a limited number of staff members who supervise qualitative dissertations in a given psychology department, identification of specific individuals is possible if institutions or exact locations are known. In the analysis section, we refer to the participants as supervisors, to highlight the focus on this aspect of their academic role in the context of this project.

\section{Analytic procedure}

A number of steps were taken to ensure a rigorous thematic analysis (Braun \& Clarke, 2006) of the transcribed interview data. The analysis was undertaken by one of the authors (SW) and two research assistants independently; the themes and extracts were then reviewed by the remaining authors to ensure reliability and validity of the analytic process. Following Braun and Clarke (2006), the analysis was undertaken as follows. The full data corpus was read repeatedly to become familiar with the content of the interviews and to identify potential areas of interest. The initial coding was then conducted using an inductive, line-by-line approach; where available, this was undertaken using MaxQDA software to help organise and highlight coded sections of the data. The codes were applied to sections of the data corpus where these related to the research question: aspects of supervision process or practices, or of support for staff or students, for example. As much as possible, the surrounding text was included in the coded sections to ensure that the immediate context of the data was preserved (Bryman, 2001). Often, sections of data were included in more than one code; for instance, discussion about 'early supervisory experiences' could overlap with 'support/guidance' as well as 'staff expertise'. The codes were 
then collated into themes, with a focus on providing a coherent analytic narrative across the dataset. The focus was on identifying patterns across the dataset: issues or ways of talking about supervision practice that featured across the participants’ accounts.

As such, the thematic analysis was conducted at the latent level; we took a broadly constructionist approach to the data in that we treated participants' accounts not as a reflection of individual motivations but as the product of sociocultural contexts (including the interview as a social context in itself). In this way, the data was not treated straightforwardly as 'fact' or 'experience' but as accounts of processes and practices, produced for the purposes of the interview. The identification and labelling of themes is thus itself a constructive process, but it can be used to identify patterns across supervisors' accounts and provide an interpretation of these in order to make recommendations for practice. That patterns and similarities could be identified across the seventeen participants - and where each participant had different backgrounds and expertise - is evidence itself of shared ways of talking about supervision and the concerns that arise therein.

In the process of refining the analysis, each theme was fitted into a hierarchical structure (with three main themes and sub-themes within each) and this was an iterative process of checking how the themes fitted together and how these related to the data corpus. For instance, the sub-theme 'second marking tensions' might have been included in the main theme of ‘supervision process' rather than the ‘quantitative culture’ main theme. What was most important was that recurrent issues raised by the participants were represented appropriately, and that there was a coherent narrative to structure the themes. Main themes and sub-themes were then labelled, and illustrative extracts used to support each sub-theme. 


\section{Analysis}

Table 2 below illustrates the main themes and sub-themes with example data extracts. In the analysis section, we further detail and discuss each theme in light of existing research in this area.

Table 2 here

\section{The 'quantitative culture’ in psychology teaching}

The first main theme presents the issue of the dominant quantitative culture within psychology teaching in relation to the skills that students possess in qualitative research methods, the relative popularity of qualitative dissertations, and the perspective of other staff members at the dissertation marking stage. The impact of the quantitative culture in psychology departments and the 'uneven playing field' identified in Gough et al.'s (2003) study over a decade ago still appears to be a dominant issue for qualitative supervision.

\subsection{Research methods teaching}

While we asked supervisors specifically about their experiences of dissertation supervision, they all referred back to the limited amount of qualitative research methods teaching at undergraduate psychology level, and the implications of this for those students undertaking a qualitative dissertation. For many of the supervisors' own departments, qualitative methods teaching may have had from as little as one or two hours' of lecture time, to a few weeks; in most cases, qualitative methods were 'tagged on' to the end of a quantitative module. As a result, it was 
argued that students often lacked a clear understanding of what is expected of a qualitative dissertation:

“They’ve had three years of general psychology and then we're asking them to prepare a piece of original research; they often have no idea how a qualitative study could be conducted” (P8).

Alongside the lack of knowledge of qualitative methods is the relative lack of importance that students (as well as some staff members) can place on qualitative approaches:

“One of the problems is that they don't get examined on qualitative, therefore they don't see it as, in any way, something they need to bother about. Someone actually told me that 'I'm not going to come to the class today because it's not something we are going to be examined on, it's not something I'm going to use'” (P4).

Students' limited knowledge and appreciation of qualitative methods was therefore noted at various points by supervisors to have an impact at all stages of the dissertation: from designing appropriate data collection methods, collecting and transcribing and analysing the data, to writing up the dissertation. A consequence of this might be that supervisors may have to spend inordinate amounts of time with students to help them grasp various issues - teaching as well as supervising research methods - and to help them through the difficult stages that they encounter. This also means that when deciding on a choice of methodology, supervisors often report staying within their comfort zone (see theme 2), suggesting methodologies with which they are familiar themselves: reflecting time, expertise and confidence concerns. Supervisors might also encourage students to take on simpler projects as a result of their lack of prior training (c.f. Shaw 
et al., 2008). The immediate impact of the limited research methods training, therefore, appears to seriously reduce the range and depth of qualitative dissertations.

Many of those supervising qualitative dissertations are also reportedly the same members of staff who teach qualitative research methods: "I’m at an institution where there is a little bit more qualitative teaching but I'm pretty much the one that does it so if I wasn't here I'm not sure what they would do" (P1). In these cases, supervisors will have a much clearer idea of what students have been taught, but this also means that students may be getting a limited perspective on qualitative methods more broadly. Their knowledge and experience could be based almost entirely on the skills and expertise of one staff member: "I think our students really have quite a narrow kind of spectrum of what qualitative work is. Just a shed load of work that they often just don’t get.” (P9). Supervisors of qualitative dissertations may therefore be isolated, with few colleagues to provide academic and methodological support, and little diversity for the students to learn different approaches or different perspectives on qualitative research.

\subsection{Rarity of qualitative dissertations}

Given the limited exposure students might have to different kinds of qualitative research methods, it is perhaps unsurprising that so few appear to take it up for their dissertation project; numbers reported by the supervisors in this study suggest that it was a minority who take on qualitative projects. For some, they may only do so if they are "number-phobic” (P2), or there is the perception of the qualitative project as an easier option. The limited numbers of students choosing a qualitative approach may also be due to the broader quantitative culture in psychology to which our students are not immune: 
"I think students also pick up through some of the kind of agitated debates you see in publications like The Psychologist that there is a little bit of an undercurrent of scepticism against qualitative methodology, and so they worry that a qualitative piece of research might be seen as inferior by some staff, so the students themselves are slightly cautious about that" (P2).

Using mixed methods may be a way of compromising these concerns: introducing students to qualitative methods while also providing the security of a statistical approach. This may still be a challenge, however, and not all supervisors might feel confident or comfortable supporting such projects. Indeed, one could argue that mixed methods could demand even more of the student and supervisor, requiring them to engage in two separate methodological stances and having sufficient competence in each to be able to complete a good dissertation.

Due to the relatively limited number of students undertaking qualitative dissertations, many supervisors noted that they lacked examples of successful dissertations that would be helpful for students, supervisors and second markers:

"I suppose what would be good would be to have dissertations with comments as well so having sort of embedded comments that say, "this is really good because they've done this, this and this” or, “this is not good because they haven’t done this”... and I think one of the things that everybody struggles with is quality and how you can determine quality from pieces of work so what differentiates a really good project from one that's not so good would be very helpful” (P11). 
Examples of this kind could help supervisors to maintain sufficient quality in a particular dissertation, to guide students on specific issues and to provide reassurance in terms of what standards they should expect of their students. Access to previous dissertations has already been noted as good practice for qualitative supervision (Gough et al, 2003; Madill et al, 2005), but many supervisors simply don't have access to these, unless they have been supervising qualitative dissertations for a number of years, and have examples of dissertations of varying quality. What would be helpful, therefore, would be open access to dissertations through a national or international repository.

\subsection{Second marking tensions}

The last sub-theme under 'quantitative culture' was perhaps the most troublesome. It relates to the final stages of a dissertation project, when the efforts of the student and supervisor are judged by, typically, two markers within the psychology department. In the processes reported in the dataset, the first marker is often the supervisor; the second marker in many cases (and especially in those departments where there is only one member of staff with qualitative research expertise) will often have no experience with qualitative methods. This has led to supervisors having to defend their students' work, not on the basis of quality, but on the basis of whether the qualitative approach is adequate. The need to justify qualitative research in psychology continues

in dissertation supervision as much as it does in research methods teaching more broadly (Gibson \& Sullivan, 2012; Hansen \& Rapley, 2008): “There are a group of colleagues who are sceptical about qualitative work. There's no question about this, there's some prejudice about qualitative work in some quarters” (P2). The exasperation of supervisors of qualitative dissertations is, understandably, apparent: 
“Perhaps it’s an over-sensitivity on my behalf I don’t know, but I just have a sense that qualitative work still has that quizzical response of, 'I don’t really understand this, I don’t know the basis for judging good or bad'... all the qualitative papers just cause confusion, they go, ‘oh I don’t know, can’t judge this’, you think, ‘Really? You can read it!’” (P9).

Whether or not the second marker lacks confidence and understanding of qualitative methods, or is prejudiced against them, the response seems rarely to be to spend some time becoming familiar with this approach in order to mark it appropriately. As noted by one participant (P15), knowing enough to judge a specific method or analytical approach is a mark of professionalism; we need to be adaptable to deal with the diversity of approaches and methods in psychology. In practice, however, time and research pressures often mean that staff members do not do this extra work to ensure they can judge the standard of different methodologies. More worryingly, what can happen is that unless the supervisor has the confidence (and authority) to be able to stand up to other colleagues, then the decision about dissertation grade may err in favour of doubt about qualitative work:

"There are colleagues in universities who will routinely try to give a poor mark to an extremely well conducted piece of qualitative research because it's qualitative research. Now how does one deal with that as a supervisor? I refuse to allow that to happen. On occasion I have made a formal complaint and insisted that a different external marker be assigned...but there's another way of dealing with it which is not to offer up qualitative dissertation theses...there could be other people who think, ‘actually, I don't need to get involved in all that kind of trouble, it would be actually much easier if I just go for attitudes’. It places on qualitative research supervisors an added burden” (P8) 
One of the main issues arising from this study, therefore, is the impact of wider research methods teaching on the number and quality of qualitative dissertations. Many departments still appear to have minimal qualitative methods teaching, and often only one or two staff members with expertise in qualitative methods in their own research. Students may then be heavily reliant on their supervisor's expertise, and even then there is a risk that the dissertation will be marked by staff members who are not familiar with the chosen methodology.

\section{Supervisors' expertise}

The heavy reliance on supervisors' expertise highlights the second main theme. Supervisors frequently referred to their own experience (or lack of) with qualitative dissertations, and of the impact of this on their availability for supervision, confidence in supporting their students, and the overall outcome of the dissertation process. This is despite the fact that most of our participants had at least 5 years' worth of supervision experience - some with much more than this - and they often had extensive expertise in using qualitative methodologies in their own research.

\subsection{Early experiences}

Whether or not the staff members had qualitative research expertise, their early experiences of supervising qualitative dissertations were characterised as being "thrown in at the deep end" (P3), like "the blind leading the blind" (P11) and "like a leap in the dark" (P17). As with many other aspects of academic life, “you were just expected to get on with it” (P14), though given that the dissertation is such an important part of a student's degree, supervisors reported being 
acutely aware of their role in this. The process was reportedly fraught with uncertainty and "as you become more experienced you become more relaxed and you're not as nervous that you're not saying the right thing” (P15).

Supervisors, even at this early stage, provided accounts of being very aware of the responsibility of this part of their role, of not wanting to 'mess up' their student's education. The concern was such that if the supervision wasn’t up to scratch:

“they'd get a rotten dissertation degree and they'd end up working in Safeway’s stacking shelves or something; that obsessed me when I was younger - I still think about it now but it obsessed me when I was young, the responsibility and the duty of care for this individual, and it still does to a large extent but I've just gradually got used to the stress level associated with it.” (P8).

One of the main ways in which supervisors dealt with the uncertainty of these first experiences of supervision was to talk to colleagues who were more experienced, whether they were close at hand (in the next office, or same department) or elsewhere (known through other networks or previous contacts). This, of course, is hugely reliant on supervisors having relevant people to ask, and if they are new to qualitative research themselves, they may not know anyone with experience in this area. There is also the potential stigma or embarrassment - as the 'new kid on the block' - to ask for help: "I think that's just a confidence thing in terms of feeling you can do that and you don’t look like an idiot, you know, if you need to ask people.” (P13). 
Without guidance from colleagues or formal training, staff members were more likely to be guided by their own individual expectations or assumptions about the supervision process. For some, this meant leaving the decision-making to the student: "I would probably be far more inclined than I am now to go with what the student wanted.” (P5). Whereas others were more idealistic in what they expected from students:

"My standards were a heck of a lot higher I think and my expectations of, of what the students would do. And I remember (name of colleague) pulling me up once about a student of hers actually, that I had helped with her analysis and apparently got quite upset just because, because I had gone through in really fine-detail her analysis. So I think, I think I have become more realistic as to what you can expect from dissertations.” (P6)

With experience and with respect to their current supervisory practice, staff members talked about being more cautious with their student dissertations, providing clearer guidance and structure for students, and setting more realistic goals and deadlines. These changes, however, appear to have developed slowly and through gradual exposure to other ways of supervising and learning from their own mistakes. For those making the move into qualitative supervision - and therefore possibly experienced in supervision, but not in qualitative supervision - the abilities and independence of the student could make all the difference. Five of our participants - those who used either only quantitative or a mix of qualitative and quantitative methods in their own research - noted that it had, in fact, been their students who had helped to support them in supervising a qualitative dissertation in the first instance.

\subsection{Absence of training and guidance}


The absence of any suitable training or specific guidance on qualitative supervision - so keenly noted in those first years of supervision - continued throughout our supervisors' accounts of their careers. This mirrors the experience of those supervising social science dissertations more broadly (Todd et al, 2006). Even for those who were experienced in using qualitative methodologies themselves, they felt ill-equipped in many aspects of their role: “I feel like I’m making it up as I go along” (P1), "just worked trial and error essentially, a lot of learning on the job” (P9). While many aspects of an academic role would benefit from experience and time in the role, with undergraduate supervision there is a risk that perceived student support will be dependent only on what the supervisor knows or has had previous experience of.

As noted in the previous sub-section, our supervisors reported being acutely aware of the importance of the supervisory relationship to undergraduates undertaking their first experience of designing and implementing original research. When supervisors discuss their approach to the process of supervising qualitative dissertations, they characterise their practices as the result of previous supervision experiences: "what did my supervisor do for me, and that sort of thing really” (P3). Although it is tempting to assume that only those supervisors with previous experience of qualitative research supervision are able to draw on their experiences to model their current practices, the notion of dissertation supervision as a practice which is developed experientially rather than through explicit training appears to cut across relative levels of previous experience in qualitative research: “It's one of these areas where you're kind of expected just to know, it’s a kind of craft skill that you've picked up over the years” (P2). 
Overall, the supervisors had somewhat fragmented training experiences; their training was by no means fully cohesive (“bits of training” and “just reading”), and largely, participants appeared to learn through their own means; that is, talking with other "colleagues who are more involved”, or through reading and personal enrolment in qualitative research workshops. In some cases, training was not even considered necessary: “I wasn’t expected to and it was just because they thought I would be a better person” (P17). The training referred to was mostly qualitative methods training, rather than the supervision of qualitative methods. In the current competitive research climate, of course, the provision of training on its own is unlikely to be a panacea for this issue: "Yeah, I suppose the truth is I need training in it and I haven’t had it, but in fairness I’ve not actively pursued it. There’s so many other things we've all got to be doing” (P10).

Being aware that you need training in qualitative supervision, and having the time that one can accountably spend on this, are two different issues entirely. Unless time and value is added to the training of supervisors in this area, it is left to the individual staff member to decide what, how, and when training should be undertaken; even then, the availability of such training either within or outwith the staff member's institution is limited. As was noted earlier, the quantitative culture still holds sway in psychology methods teaching, and this has ramifications not only for students, but also for supervisors as well.

Supervisors' talk about the absence of recognised and demarcated training for supervising (qualitative) dissertations reveals a tension between the public practice of teaching in classroom environments which can, and is, observed as part of professional development and the "private realm” (P1) of supervision. As noted earlier, supervisors may not feel confident in seeking help 
or informal support from their colleagues for fear of looking "like an idiot" (P13), or there may be a lack of colleagues nearby who can help, unless “you're lucky enough to have a colleague who works in that area then you can maybe get some feedback from” (P14). The relatively private realm of the supervisor-student relationship means that there may be fewer opportunities, and no formal mechanism to discuss supervision issues with others; as noted by Rowley and Slack (2004), the supervision process is opaque. When talking about support needs that might be useful, therefore, many of the supervisors referred to a resource - such as an online resource that could be used by both students and supervisors, as they go through the dissertation process together. Rather than a book which might just sit on the shelf, an online space could provide a source to dip into at various points, for access to materials or a space where supervisors and students could share experiences, answer questions and offer help: "helping students get through particular points that they're stuck on or helping students to deal with things like 'everyone else is doing quantitative and I'm doing qualitative', how do you deal with challenging questions" (P1). Since not all supervisors have access to informal support or guidance from colleagues, an online resource could provide a space in which supervisors and students could share questions and answers on specific topics.

Supervisors also illustrated the importance of having a sense of support and of relevant contacts ‘being there' as a means of bolstering supervisory confidence or “airing frustrations” (P6), rather than explicitly providing advice to one another. There is an informality behind peer and supervisory interaction and support; it is typically only sought when the need arises, a resource that can be drawn upon as problems or issues occur. For example, by stating that they would 'discuss' the problems illustrates that solutions were not achieved through sole reliance on 
another individual, but came as a result of active engagement and interaction with a peer, to someone who is "talking the same language” (P7) in terms of qualitative methods. Therefore, it could be argued that supervisors require a support-based system where they can not only solve problematic situations, but also vent their frustrations with others in a similar position.

\subsection{The comfort zone}

As a consequence of the lack of training and guidance in the supervisory role, our supervisors reported feeling more comfortable (“on safe ground”, P1) and able to supervise only those methodologies that they use themselves. Already this places limits on what options might be available to students; though as noted earlier, if the student is determined and competent enough, they may be able to persuade the supervisor to take on a new approach. In most cases, however, it is the supervisor who has the final say over which methodology is used:

“I would do some reading myself. I wouldn’t probably encourage a student to use a method that I wasn’t- that I didn’t know anything about, so I'm hoping that my expertise will be at least equal to that. I never feel as confident supervising a qualitative project as I would [a quantitative one] because I think there are no right or wrong answers with a qualitative project” (P11).

Feeling confident and experienced enough to supervise a particular methodology also meant that supervisors often constructed it as being more fair or beneficial to the students if they supervised an approach that they had experience in; they would be able to provide better support because of their expertise. This was noted both about making a shift from quantitative to qualitative, as well as making judgements about different qualitative methodologies (such as phenomenological 
rather than thematic analysis). This might not always be the case, though, as a few supervisors noted that because of their expertise they were less able to see the data in the same way that the students did. They had almost become too expert in that the distance between their knowledge and the student's knowledge was much greater, and this could arguably be daunting and offputting for the students.

Supervisors also highlighted the need to be validated by other 'experts’ within qualitative research, and this was particularly the case for those who had a background in quantitative research and who talked about “dabbling in” (P2) or making a "scary shift” (P7) to qualitative work or being an “imposter” (P7, P10) because they don’t have an extensive background:

“I think I’d always feel more comfortable having a - someone with more qualitative expertise involved either as a supervisor or to run some stuff by them; I just feel that - I feel an imposter doing it solo... I want to differentiate between a true, outstanding qualitative work and- yeah I'm not sure that I'm a good judge of that.” (P10)

This is troubling in itself; the supervisors were downplaying their own expertise even within the interview setting, and oriented to the need to feel "legitimate” (P7) or be an expert in order to supervise qualitative dissertations effectively: “I haven’t, I must confess, been formally trained in qualitative methods” (P2), “I wouldn’t call myself really a qualitative researcher” (P11), “I usually take advice from an expert in the field” (P13). Given that those who do have a history of using qualitative research also noted that they felt underprepared for the supervision process, or not competent to supervise all qualitative approaches, it follows that experience in using these methodologies is not sufficient in itself. 
Similarly, having credibility amongst the students was also regarded as important (cf. Calvert \& Casey, 2004). Given the status of qualitative research in their research methods training more broadly, and the variety of approaches that supervisors can take with qualitative research:

“Everyone will approach it in a slightly different way and it’s not that one way is right and one way is wrong but I always think that if the student hears from other people they have to have the faith in you as their supervisor that what you're getting them to do is okay” (P14).

The second theme has therefore illustrated how reliant the supervision process is on the specific experience a supervisor has in a particular area. Due to the nature of qualitative research, which varies considerably in terms of epistemological, theoretical and methodological grounds, no supervisor claimed to be competent in all qualitative methods, even if they had extensive (over 20 years’) experience in qualitative research themselves. They mostly supervised in their 'comfort zone': those methods which they use themselves, or have a little knowledge of. With no formal training or guidance in this area, the support for qualitative supervision - both for students and supervisors - is dependent on whoever happens to be available in that psychology department, in that university.

\section{Supervision process}

The final main theme relates to the supervision process, from the allocation of students to supervisors, to progression through different stages of the qualitative research. As will be seen, 
this theme is interwoven with the earlier two in that decisions made at each stage depend largely on supervisors' expertise and confidence in the student, as well as understandings of the qualitative methodology and its suitability for the dissertation project.

\subsection{Matching supervisor and student}

While previous research (Rowley \& Slack, 2004) noted that, in practice, supervisors may find themselves working with topics and methods outside of their expertise area due to the allocation process, we found that the opposite was more likely to be the case with the supervisors undertaking qualitative dissertations. Supervisors were more likely to stay within their comfort zones (in terms of methodologies supervised), and many departments were becoming more prescriptive over the choice of projects for students. Supervisors may suggest topic areas, specific research questions, or even clearly defined projects, from which students can choose to best match their interests: "the reason being just generally students were finding it very hard to get going and to identify a question, and therefore the dissertations would be substantially delayed or not as good as they could be” (P6). Being prescriptive in this way means that students may have a better chance of undertaking a 'do-able’ project, but less freedom in terms of their own research interests. Students were also being protected, as seen in masters-level supervision (Shaw et al., 2008), from the relative risks of doing a qualitative project, whether this was through the selection of a 'simpler' qualitative methodology, or the restriction to a quantitative project only (cf. Gough et al., 2003).

The practical arrangements for allocating students to supervisors varied considerably between institutions in our sample, ranging from emails and online group selection to students knocking 
on supervisors' doors. The somewhat chaotic manner in which students might be allocated to supervisors also has implications for the choice of methodology that might be studied and the projects undertaken, as well as students’ degree of ownership and responsibility for their dissertation. In many institutions, whether or not students were able to secure their preferred supervisor/topic was often a result of a 'first come, first served' process, where the students who were quickest to email, electronically sign up to, or find the door of, the supervisor were those who had first choice. While in some cases, supervisors might refuse to take on a student, or a student may find another supervisor, in practice many systems don’t allow for such flexibility. If other supervisors have no spare 'supervision spaces', for example, then students do not have the choice to move after the initial allocation process.

The implications of this for the project methodology become apparent at the next stage: in the initial meetings between supervisor and student when they agree on a topic, a research question, and the appropriate methodology. As was noted earlier, students often approach supervisors with limited knowledge of qualitative methodologies, so the delicate management of matching up a 'do-able’ project (on which the supervisor can offer some guidance) with an appropriate methodology and the student's own capabilities, then becomes condensed into what may only be a couple of short meetings or email exchanges between student and supervisor. As noted earlier, many supervisors felt able to only supervise those qualitative methodologies with which they had some experience or expertise; staying within their comfort zone for their own, as well as the students', benefit. While the choice of methodology should in principle follow the topic and research question that the student wishes to study, therefore, in practice it is more heavily guided 
by the supervisor's own expertise and their assessment of how capable the student might be (cf. Todd et al., 2006).

\subsection{Progression and student support}

The nature of qualitative research means that it typically has a different trajectory to quantitative research, and can often take longer at different stages. Alerting students to these different timescales can be a major focus for supervisors: "I'm very much more aware of kind of deadlines, how more organised we need to get the students” (P12). While students using quantitative methods may be able to allocate a week or two to the analysis stages, it can be difficult to persuade students just how long transcription, coding and analysis can take. A considerable amount of time, therefore, is spent preparing for, and dealing with, underestimations of time and effort needed for qualitative dissertations:

"I guess quite often I am cautious about students doing qualitative work in part because of time investment that it takes, the time on their behalf which I think- my experience has always been they massively underestimate it - than time actually being able to work with them.” (P9)

This in turn links back to the decisions supervisors make when they first meet with a student: whether to take on their project and whether the student is capable of doing a qualitative dissertation. Additional time might also need to be factored in to collect additional data, particularly if the students are using interviews and have no experience of conducting these:

"I always get them to do one pilot interview, transcribe it, bring it, and then we sit down together and go through it and work out do we need to adjust the interview schedule and 
nine times out of ten we do. And that's ...they don't get it until they have done the interview and then they've looked back and they go 'huh, I should have asked that...why didn’t I ask that?' or even if they don’t realise the questions aren’t appropriate.” (P14)

An awareness of deadlines, therefore, was considered essential, both for the student and the supervisor. Despites this, supervisors often had to manage the highs and lows of qualitative dissertations and what one supervisor described as the 'horse race' between those doing quantitative and those doing qualitative dissertations. At different points one student might be further ahead (data collection), but then quickly fall behind at the next hurdle (data analysis). This can lead to various points of "genuine panic about the messiness of qualitative data" (P8). Having an understanding of the stages at which students might panic or be concerned is therefore crucial, as is the awareness that while most qualitative data is potentially analysable (in contrast to, say, having an insufficient number of participants to conduct a statistical analysis), not all qualitative data will produce a good dissertation or analysis.

As a result of the quantitative dominance in psychology dissertations, and the different trajectory of qualitative projects, supervisors reported being acutely aware of the need to provide additional support for their students and used various strategies such as students working in pairs for their dissertation (on the same topic, with the same data, but different research questions), offering group supervision meetings, and offering more frequent, or longer, supervision sessions with their students. Student support, however, like supervisor training and resources, "feels a bit haphazard at the moment” (P7), and often creates additional workload and concern for supervisors. There were also concerns that “there's a slight ambiguity as to what they can expect 
from their supervisor” (P1), and offering support while also encouraging the student to maintain responsibility and control for their project. Being supportive could, if not effectively managed, mean taking charge of a project and preventing students from gaining experience of the different stages of qualitative research.

\section{Discussion}

The results from this study echo, and provide further empirical support for, the limited research that has been conducted around undergraduate supervision in the social sciences more broadly (Rowley \& Slack, 2004; Todd et al., 2004, 2006) and qualitative psychology supervision specifically (Gough et al., 2003; Madill et al., 2005). Of concern is that some key aspects of the situation noted over a decade ago appear largely unchanged: the supervision of qualitative dissertations is still challenging due to the limited prior training of students in qualitative methods at undergraduate level (at least in our sample of Scotland/North East England) and the dominant quantitative culture reported within these psychology departments. The supervisors we interviewed also reported a lack of confidence when supervising methods that they don’t themselves use. As confidence in one’s supervisor may be crucial for student's perception of their own dissertation (Calvert \& Casey, 2004; Todd et al., 2006), there is a need to provide support or training for those who are supervising methods with which they are unfamiliar.

There are, however, small signs of change. Qualitative methods teaching is gradually developing, and as the number of staff members experienced in qualitative methods increases, students will have more opportunities to undertake a qualitative dissertation and thus broaden the skill base of psychology graduates in line with subject benchmarks. The current generation(s) of supervisors 
are also likely to have had a predominantly quantitative research methods training background in psychology themselves, and this trickles down to their supervision of undergraduates. It follows, then, that as qualitative methods are taught more thoroughly across undergraduate and postgraduate psychology courses, that subsequent generations of supervisors will be better equipped.

Furthermore, while the supervision process is still ‘opaque’ (Rowley \& Slack, 2004), we hope to have shed some light on the processes and practices of supervision of qualitative dissertations as described by supervisors themselves - and in doing so, provide reassurance for others who may be in similar situations. The message from this project is clear: there were common patterns across supervisors' experiences in psychology departments in Scotland and North East England, and these provide further empirical support for the earlier research in this area. As supervisors ourselves, we can also offer some reflection on this research project. Conducting the interviews and analysis provided a rare opportunity for us to talk to colleagues (some we knew, some we didn't know prior to the research) about an issue that is seldom discussed at length in our daily routines. We were also aware of our own expectations and assumptions around supervision, and while we cannot claim to be 'neutral', ensured at least that we allowed our participants' accounts to take precedence and to include examples from every interview to illustrate the thematic analysis. The themes presented here are, by definition, a constructed narrative about supervision practice of qualitative dissertations, but we hope to have provided a coherent argument that shows consistency across the dataset and a clear vision for where this might take us next. 
As such, we argue that there is a pressing need for training and guidance in qualitative dissertation supervision in psychology, and for practical resources for both staff and students. To this end, one of the main recommendations of this study is the need for a freely accessible, online resource that could provide guidance and informal support for students and supervisors/second markers, and might include some of the following features:

- Examples of marked dissertations using different qualitative methodologies, with notes that indicate the strengths and weaknesses of the dissertation (cf. Gough et al., 2003; Madill et al., 2005).

- Links or direct access to various tools and materials for conducting qualitative dissertations, such as transcription, coding and/or analysis software and examples of data (such as the TQRMUL friendship interview corpus available at http://bit.ly/TQRMULdataset).

- Blogs, case studies or testimonials from students and supervisors on specific issues related to qualitative dissertations.

- A forum where students/supervisors can ask questions and offer replies to each other using asynchronous discussion, to allow support as and when it is needed.

- Suggestions about timelines and progression within qualitative dissertations, to help students and supervisors to plan their studies effectively.

The online resource would provide an accessible, interactive, and evolving means of dealing with issues as they are dealt with nationally and internationally. This would be particularly invaluable for isolated students and supervisors, to provide not only practical support but also a means of sharing concerns and solving problems. 
In addition to the online resource, other recommendations from this project are:

- The need for psychology departments to have, where possible, more than one staff member with expertise in one or more qualitative methodologies. This would not only provide support for teaching and supervision, but would also be a further form of expertise for second marking.

- The need for training in undergraduate supervision more broadly, and qualitative supervision in particular. Due to the increasing range of qualitative methodologies, even supervisors who use qualitative approaches in their own research will have gaps in their knowledge and expertise. Such training might be in the form of interactive, peerdiscussion workshops, to share good practice and learn strategies for effectively supporting qualitative dissertations. While similar suggestions have been made elsewhere (Maunder et al., 2012), it appears that they have not been broadly taken up.

- The need for greater coverage of qualitative research methods teaching at undergraduate level, and for a clearer integration of this with quantitative methods: as parallel endeavours, rather than qualitative as an add-on at the end of a statistics module. This might also include greater resourcing of qualitative methods teaching, such as the provision of software and video recording devices (Mitchell et al., 2007).

This study has provided up-to-date reflections on the current processes and practices of qualitative dissertation supervisors in psychology departments across North East England and Scotland. While the themes were consistent across the data corpus (17 supervisors), there are limitations in that experiences of supervisors in the rest of the UK may be different, and this may 
reflect different degree structures (three years in England rather than four years in Scotland) and the introduction of student fees across English universities. We hope to have provided, however, a glimpse into the 'private realm' of supervision from the perspective of the supervisors, and in doing so, to offer reassurance to those who may be in similar positions, and to provide concrete recommendations for how support and guidance may be developed in this area.

\section{Acknowledgements}

The authors would like to thank Gillian Hendry, Ryan Kelly, Robert McQuade and Kim Singh for their research assistance at various stages of the research project. We are also grateful for financial support for this project, which was received from the University of Strathclyde's Education Excellence Fund and Teeside University's Teaching fund. We would also like to acknowledge the support of members of the BPS QMiP Section’s Teaching Qualitative Psychology Group, formerly the Teaching Qualitative Psychology (TQP)/Teaching Qualitative Research Methods at Undergraduate Level (TQRMUL) Special Interest Group of the Higher Education Academy.

\section{References}

Anderson, C, Day, K, and McLaughlin, P 2006, 'Mastering the dissertation: lecturers' representations of the purposes and processes of Master's level dissertation supervision', Studies in Higher Education, vol. 31(2), pp. 149168.

American Psychological Association 2010 Publication manual of the American Psychological Association (6th edition, APA, Washington, DC.

Braun, V and Clarke, V 2006, 'Using thematic analysis in psychology,' Qualitative Research in Psychology, vol. 3, pp. $77-101$. 
Bryman, A 2001, Social research methods, Oxford University Press, Oxford.

British Psychological Society 2014, Standards for the Accreditation of Undergraduate, Conversion and Integrated Masters Programmes in Psychology, BPS, Leicester.

Calvert, B and Casey, B 2004, 'Supporting and assessing dissertation and practical projects in media studies degrees: towards collaborative learning,' Art, Design and Communication, vol. 3(1), pp. 47-60.

Castle, P, Incledon, V, and Waring, M 2008, 'Enhancing feedback: the development of a criterion- based marking template for students on a sport degree programme,' Journal of Hospitality, Leisure, Sport and Tourism Education, vol. 7(1), pp. 89-98.

Cooper, MJ 2001, 'Evaluating a D. Clin. Psych. research dissertation proposal: qualitative studies,' Clinical Psychology, vol. 2, pp. 21-24.

DeAngelis, T 2010, Closing the gap between practice and research. Retrieved 19/08/2013, from http://www.apa.org/monitor/2010/06/gap.aspx - See more at: http://reffor.us/index.php\#sthash.9cIrnyJn.dpuf

Forrester, M.A. \& Koutsopoulou, G. Z. (2008). Providing Resources for Enhancing the Teaching of Qualitative Methods at the Undergraduate Level: Current Practices and the Work of the HEA Psychology Network Group, Qualitative Research on Psychology, vol. 5(3), pp.173-178.

Gibson, S and Sullivan, C 2012, 'Teaching qualitative research methods in psychology: an introduction to the special issue,' Psychology Teaching and Learning, vol. 12(1), pp. 1-5.

Gough, B, Lawton, R, Madill, A and Stratton, P 2003, Guidelines for the supervision of undergraduate qualitative research in psychology (No. 3), LTSN Psychology, York.

Hansen, S, and Rapley, M 2008, Editorial: Special issue of qualitative research in psychology on 'teaching qualitative methods'. Qualitative Research in Psychology, vol. 5(3), pp. 171-172.

Harper, D J, O'Connor, J, Self, P, and Stevens, P 2008, 'Learning to use discourse analysis on a professional psychology training programme: accounts of supervisees and a supervisor,' Qualitative Research in Psychology, vol. 5(3), pp. 192-213

Heinze, A, and Heinze, B 2009, 'Blended e-learning skeleton of conversation: improving formative assessment in undergraduate dissertation supervision,' British Journal Of Educational Technology, vol. 40(2), pp. 294-305. 
I'Anson, RA. and Smith, KA 2004, 'Undergraduate research projects and dissertations: issues of topic selection, access and data collections amongst tourism management students,' Journal of Hospitality, Leisure, Sport and Tourism Education, vol. 3(1), pp. 19-32

Lees, J 2008, 'Research supervision and psychotherapeutic theory: bridging the research-practice gap,' Psychodynamic Practice, vol 14(3), pp. 281-293.

Madill, A, Gough, B, Lawton, R, and Stratton, P 2005, 'How should we supervise qualitative projects?' The Psychologist, vol. 18(10), pp. 616-618.

Maunder, RE, Gordon-Finlayson, AR, Callaghan, J and Roberts, A 2012, Behind supervisory doors: Taught Masters dissertation students as qualitative apprentices. Psychology Learning \& Teaching, vol 11(1), pp.30-38.

Maxwell, TW, and Smyth, R 2010, 'Research supervision: the research management matrix,' Higher Education, vol. 59(4), pp. 407-422.

Mills, C, and Matthews, N 2009, 'Review of tutor feedback during undergraduate dissertations: a case study,' Journal of Hospitality, Leisure, Sport \& Tourism Education, vol. 8(1), pp. 108-116.

Mitchell, T, Friesen, M, Friesen, D, and Rose, R 2007, 'Learning against the grain: Reflections on the challenges and revelations of studying qualitative research methods in an undergraduate psychology course,' Qualitative Research in Psychology, vol. 4(3), pp. 227-240.

The Quality Assurance Agency for Higher Education 2010, Subject Benchmark Statement: Psychology, QAA, Gloucester.

Rowley, J, and Slack, F 2004 'What is the future for undergraduate dissertations?’ Education \& Training, vol. 46(4), pp. 176-181.

Shaw, RL, Dyson, PO, and Peel, E 2008, 'Qualitative psychology at M level: a dialogue between learner and teacher,' Qualitative Research In Psychology, vol. 5(3), pp. 179-191.

Sullivan, C, Gibson, S \& Riley, S. (Eds) 2012, Doing your Qualitative Psychology Project. Sage Publications: London. 
Todd, MJ, Smith, K, and Bannister, P 2006, 'Supervising a social science undergraduate dissertation: staff experiences and perceptions,' Teaching in Higher Education, vol. 11(2), pp. 161-173. 